Research Article

\title{
Location-Price Game in a Dual-Circle Market with Different Demand Levels
}

\author{
Xiaofeng Chen $\mathbb{D}^{1,2}$ Qiankun Song $\mathbb{D}^{1,2}$ Luqing Rong $\mathbb{D}^{1,3}$ and Zhenjiang Zhao $\mathbb{D}^{4}$ \\ ${ }^{1}$ School of Economics and Management, Chongqing Jiaotong University, Chongqing 400074, China \\ ${ }^{2}$ Department of Mathematics, Chongqing Jiaotong University, Chongqing 400074, China \\ ${ }^{3}$ School of Economics and Management, Guangxi University of Science and Technology, Liuzhou 545006, China \\ ${ }^{4}$ Department of Mathematics, Huzhou University, Huzhou 313000, China
}

Correspondence should be addressed to Xiaofeng Chen; xxffch@126.com

Received 13 September 2020; Revised 2 October 2020; Accepted 16 March 2021; Published 26 March 2021

Academic Editor: Sanbo Ding

Copyright (c) 2021 Xiaofeng Chen et al. This is an open access article distributed under the Creative Commons Attribution License, which permits unrestricted use, distribution, and reproduction in any medium, provided the original work is properly cited.

\begin{abstract}
This paper researches a location-price game in a dual-circle market system, where two circular markets are interconnected with different demand levels. Based on the Bertrand and Salop models, a double intersecting circle model is established for a dual-circle market system in which two players (firms) develop a spatial game under price competition. By a two-stage (location-then-price) structure and backward induction approach, the existence of price and location equilibrium outcomes is obtained for the location game. Furthermore, by Ferrari method for quartic equation, the location equilibrium is presented by algebraic expression, which directly reflects the relationship between the equilibrium position and the proportion factor of demand levels. Finally, an algorithm is designed to simulate the game process of two players in the dual-circle market and simulation results show that two players almost reach the equilibrium positions obtained by theory, wherever their initial positions are.
\end{abstract}

\section{Introduction}

The scale of the coronavirus disease 2019 (COVID-19) pandemic is unprecedented, which is the current challenge of all global actors. From the economic perspective, the current pandemic has had a profound impact on the supply chain, domestic and foreign demands, enterprise decision-making, consumer purchasing power, and many other aspects. For instance, New Zealand fishermen, who had already harvested more than 100 tonnes of live rock lobster for the international market, reluctantly accept the fact that the demand for lobster had dropped sharply due to the sudden cancellation of export orders. To help the economy recover from the effects of the COVID-19 pandemic, governments adopt more precise and welltargeted fiscal policies to stimulate domestic demands, boost social consumption, and investment needs. Thus, firms will face different demand levels in the international and domestic markets. Moreover, this difference in demand level will change with the recovery of the economy.
In this paper, we consider the location problem of two competitive firms in the international and domestic markets with different demand level. Taking into account the fact that each firm may adapt its subsequent actions based on the other side's actions, it is natural to turn to game theory for ideas and principles to analyze this location problem. Since game theory was pioneered by John von Neumann in 1928 [1], it has been widely applied in politics, military strategy, economics, biology, computer science, and many other fields [2-5]. Meanwhile, the location game, as an important topic in the fields of industrial organization and transportation economics, has been attracting much attention from many researchers [6-8].

From the existing literature, according to firms' competition patterns, local game models can be divided into two classical categories. One is location-quantity game and another is location-price game. Both of them are two-stage game, in which, at the first stage, each firm determines its own location. However, at the second stage, each firm in location-quantity case behaves as the Cournot oligopoly and 
conducts the quantity competition, while, in location-price case, each firm performs as the Bertrand oligopoly and develops the price competition. It should be noted that Bertrand pattern shows some more differences compared with the Cournot pattern. At the second stage of the location-price game, instead of choosing to supply a quantity for each customer in the market, each firm sets a suitable price for all customers and takes the advantage of price and distance differences to attract more customers, who will always buy one unit of the product [9]. Therefore, a Nash equilibrium occurs at this second stage when both firms do not wish to adjust their price based on the price set by the other firms, implying that their profits cannot be Pareto improved by changing prices. Generally, the price of a product is a main factor of the demand for it, while the quantities supplied in a market seem to be a more indirect factor of the demand [9]. Thus, the present research will adopt the assumption of competition in prices.

In addition to competition pattern, location space is one of the important components of location game. The type of location space directly determines the measure method of the minimum transportation distance. For instance, the minimum distance is the Euclidean distance in a convex region and the Hamiltonian distance on a grid, whereas it is the length of an arc on a circle. Different location game models with various location spaces are available in the literature [10-13].

The basic location space is the bounded linear area, which is first introduced to location game by Hotelling [14]. Inspired by Hotelling model, a great amount of rich literature of location game with linear location space was emerged. In [15], by using quadratic transportation costs in place of linear ones, a modified version of Hotelling's example was proposed to show that the principle of minimum differentiation is invalid. In [16], in the case of modifying some assumptions for the classic Hotelling model, the authors considered duopoly and spatial competition and proved that the social optimal positions of $n$ firms are the same as the equilibrium positions in a bounded interval. In [17], the author proved the existence of a Nash equilibrium in prices and locations in learn markets and examined the effects of the magnitude of changes in marginal costs and of the freight rates for one or both firms. In [18], the authors investigated the problem of existence of equilibrium prices and locations in the extended Hotelling model. In [19], the author re-examined Hotelling model of duopolistic competition and claimed that the principle of minimum differentiation is never exhibited by the subgame-perfect equilibrium. In [20], the authors made some comparisons between Bertrand and Cournot competitions in location game, where the location space is a linear bounded area. In [21], the author derived some sufficient conditions of the existence of equilibrium outcome for Hotelling games, which are dependent on the number of firms.

In the real world, markets are usually located along complex transportation networks. In order to accurately represent actual markets, a large number of researchers considered the complex location spaces such as circles, spokes, and networks. In [22], the existence and uniqueness of a unique price equilibrium in multiplayer location game are proved under the quadratic transportation cost function, where the location space is a circular road. In [23], the problems of existence and nonexistence of an equilibrium were studied for a location-price game in the circle market. A linear and circular model with spatial Cournot competition was explored in [24], where the dependence between location equilibrium and demand density was examined. In [25], the authors demonstrated that the unique equilibrium location is in the equidistant pattern for the multiple players in a circular market. The spatial Cournot competition of two identical firms was researched in two intersecting circular markets in [26], and it is proved that the unique symmetric equilibrium is located at the two intersection points. In [27], the authors considered the nonlocalized spatial competition using a spokes model and analyzed the influence of the number of firms on the equilibrium price. In [28], the authors focused on methods of computing equilibria for multisupplier location competition, where the cost structure is heterogeneous, and the location space is a network. In [29], the author researched a noncooperative location game for two mobile firms in a market region, where the location space is a subset of the plane. The results show that firms will never locate at the same time if they have the same cost rate.

Strongly motivated by the above discussion, in this paper, we investigate the two-player location-price game in a dual-circle market. The location space is considered on two intersecting circles, which can be viewed as domestic and international interconnected markets as shown in Figure 1. In such a location game, two firms choose the optimal position in the domestic market as their locations and develop price competition such that their profits are maximized. Different from the previous works [26], we consider the spatial Bertrand competition of two firms in two intersecting circular markets, where the demands of the two markets may be at different levels due to external shocks. The main contributions of this paper are highlighted as follows:

(1) Not only is the existence of a location equilibrium in the location-price game proved for a dual-circle market, but also the equilibrium outcome is presented by algebraic expression, which can be computed directly.

(2) An algorithm is designed to simulate the competitive process in which two players move dynamically in the market. The simulated and theoretical results are in good agreement with each other, which shows the feasibility and effectiveness of the algorithm.

(3) The effects of the difference degree of demand level between two circular markets on the location equilibrium are researched, which implies that the equilibrium locations of the players tend to be the maximum differentiation, if the demand level ratio of domestic market to international market is sufficiently high. 


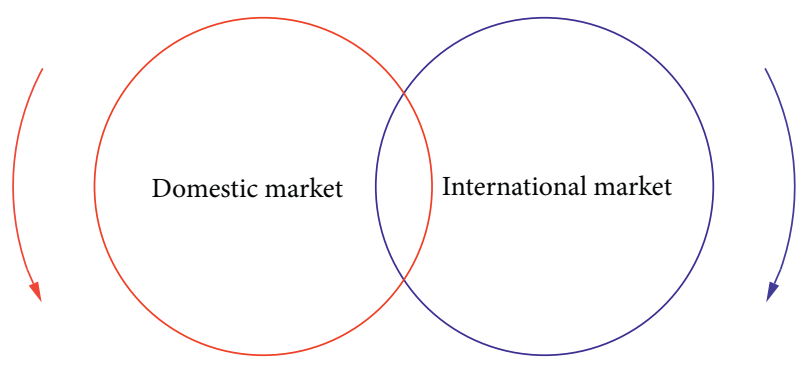

Figure 1: A dual-circle market system.

\section{Descriptions of the Dual-Circle Market Model}

2.1. Some Assumptions. There are two players (firms 1 and 2 ), which produce homogeneous products, in a dual-circle market system including domestic and international markets (as shown in Figure 1). The two players will choose optimal locations on the domestic market ${ }^{1}$ and play Bertrand-Stackelberg game to maximize their respective profits.

Let two intersecting circles (denoted by " $L$ " and " $R$ ") be domestic and international markets (as shown in Figure 2). Without loss of generality, it is assumed that the circumferences of the circles are both 1, and they intersect at two points $H_{1}$ and $H_{2}$, which can be viewed as two transportation hubs. On circle $L$ and on circle $R$, the lengths of the arcs $\widehat{H}_{1} H_{2}$ are both $s \in((1 / 6),(1 / 2)) .^{2}$ To represent the positions of the points on the circles, we map the points on circle $L$ with counter-clockwise direction and on circle $R$ with clockwise direction to the interval $[0,1]$, respectively. Assume that the leftmost point on circle $L$ corresponds to zero, while the rightmost point on circle $R$ corresponds to zero. Therefore, if the position value of point $C$ on circle $L$ is $x \in[0,1]$, it means that the distance from $O$ to $C$ on circle $L$ with counter-clockwise direction is $x$. No matter whether from the viewpoint of circle $L$ or circle $R$, the position values of $H_{1}$ and $H_{2}$, respectively, are

$$
\begin{aligned}
& h_{1}=\frac{1}{2}-\frac{s}{2}, \\
& h_{2}=\frac{1}{2}+\frac{s}{2} .
\end{aligned}
$$

Customers are distributed over circles $L$ and $R$ with a constant density $\rho$. Then, the total number of customers on each circle is $\rho$, since the length of each circle is 1 . Affected by some exogenous factors, the number of effective customers may be lower or higher than that under normal circumstances. The relationship between effective customer densities and normal customer densities can be expressed as follows:

$$
\begin{aligned}
& \tilde{\rho}^{L}=\mu^{L} \rho, \\
& \tilde{\rho}^{R}=\mu^{R} \rho,
\end{aligned}
$$

where $\widetilde{\rho}^{L}$ and $\widetilde{\rho}^{R}$ are effective customer densities on circles $R$ and $L$, respectively; $\mu^{L}$ and $\mu^{R}$ are corresponding effective customer levels. If $\mu^{L}>1$, the consumption of customers is stimulated. If $\mu^{L}<1$, the consumption of customers is restrained. Let $\theta=\left(\mu^{L} / \mu^{R}\right)$ be the proportion factor of domestic and international demand levels, which is used to measure the difference between the two demand levels.

Suppose that each firm prices the products, where the products from one of the firms are sold for the same price to any customer who then has to pay the transportation costs. Let $p_{i}$ be the mill price of the products from firm $i$. Furthermore, $T^{L}(d)$ and $T^{R}(d)$ are the transportation cost functions from circles $L$ and $R$ to the locations of firms, which are described as follows:

$$
T^{L}(d)=k\left(d-\frac{1}{2} d^{2}\right), \quad T^{R}(d)=k d,
$$

where $k>0$ is a transportation cost constant and $d$ is the transportation distance. It should be noted that the maximum distance between any two positions on the two circles is $1-s$, which is not greater than 1 . The convex function $T^{L}(d)$ is increasing on $[0,1]$ and the derivative $\left(T^{L}(d)\right) /$ is decreasing, which reflects the effect of scale transportation. Compared with $T^{L}(d)$, the transportation cost $T^{R}(d)$ from circle $R$ has no quadratic term $-(1 / 2) d^{2}$, which means that $T^{R}(d)$ is larger than $T^{L}(d)$.

The net utilities of a customer at $x$ on circles $L$ and $R$ buying products from firm $i$ are supposed as follows:

$$
\begin{aligned}
& u^{L}\left(x, p_{i}, x_{i}\right)=u-p_{i}-T^{L}\left(d_{\min }\left(x, x_{i}\right)\right), \\
& u^{R}\left(x, p_{i}, x_{i}\right)=u-p_{i}-T^{R}\left(d_{\min }\left(x, x_{i}\right)\right),
\end{aligned}
$$

where $u$ is a large positive constant representing the utility directly obtained by customers consuming homogeneous products, and $d_{\min }\left(x, x_{i}\right)$ is the minimum distance from customer position $x$ to location $x_{i}$ of firm $i$. To discuss the mathematical expression of $d_{\min }\left(x, x_{i}\right)$, we introduce a bivariate function:

$$
g(x, y)=\min \{|x-y|, 1-|x-y|\}=\frac{1}{2}-\frac{1}{2}|1-2| x-y|| \mid,
$$

which depicts the minimum distance between any two positions $x$ and $y$ on a circle with length of 1 . If customer $x$ is on circle $L$, the minimum distance can be written as

$$
d_{\min }\left(x, x_{i}\right)=g\left(x, x_{i}\right)=\frac{1}{2}-\frac{1}{2}|1-2| x-x_{i}|| .
$$

If customer $x$ is on circle $R$, the minimum distance can be written as

$$
d_{\min }\left(x, x_{i}\right)=\min \left\{g\left(x, h_{1}\right)+g\left(h_{1}, x_{i}\right), g\left(x, h_{2}\right)+g\left(h_{2}, x_{i}\right)\right\},
$$

where $h_{1}=(1 / 2)-(s / 2)$ and $h_{2}=(1 / 2)+(s / 2)$, which are position values of transportation hubs $H_{1}$ and $H_{2}$.

2.2. Range of Equilibrium Locations. Let $x_{1}^{*}$ and $x_{2}^{*}$ be Nash equilibrium locations of players 1 and 2 on circle $L$. Without loss of generality, we assume that $x_{1}^{*} \leq x_{2}^{*}$. The dual-circle market model is fully symmetrical with axis OOI in two 


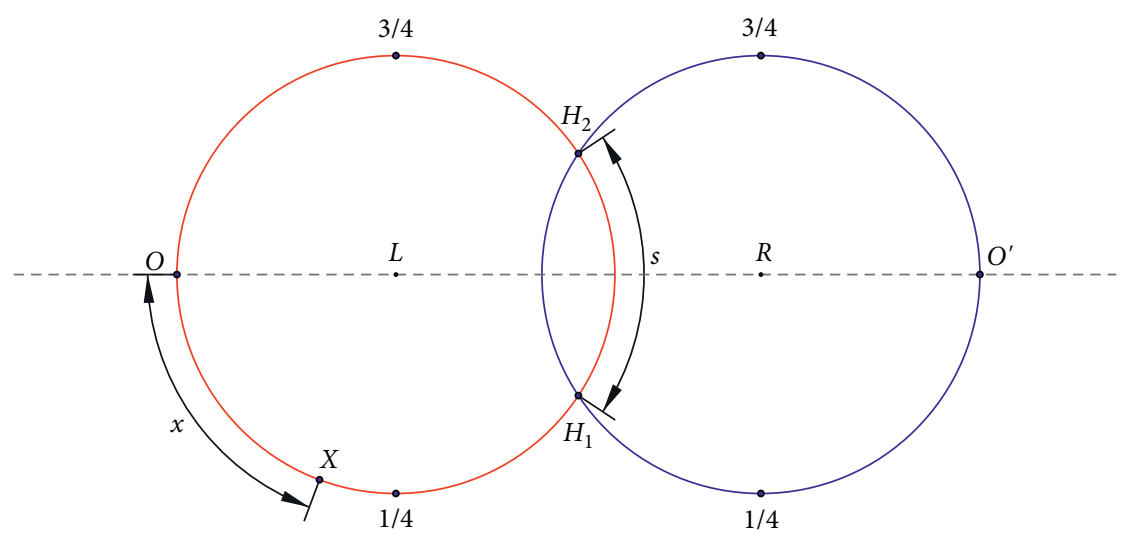

Figure 2: A dual-circle market model.

senses: (i) the geometry of the model is symmetrical, and (ii) the effective customer densities are equal at any two positions, which are symmetrical with axis. Facing such a symmetrical dual-circle market, the two players will adopt peer-to-peer strategies. Then the equilibrium locations $x_{1}^{*}$ and $x_{2}^{*}$ should be symmetrical with axis OO . In other words, $x_{1}^{*}$ and $x_{2}^{*}$ satisfy $x_{1}^{*}+x_{2}^{*}=1$. Consider two extreme situations. In the first situation, suppose that the effective customer density is zero on circle $R$, which means that the demand for products is nought. There is only one circle $L$ in the market. According to the principle of maximum differentiation for one circle market [30], the equilibrium locations $x_{1}^{*}$ and $x_{2}^{*}$ should be at positions (1/4) and (3/4). In the second situation, suppose that the effective customer density is zero on circle L. The equilibrium locations $x_{1}^{*}$ and $x_{2}^{*}$ should be set as close to points $A$ and $B$ as possible to attract more customers on circle R. Consequently, $x_{1}^{*}$ and $x_{2}^{*}$ are equal to $(1 / 2)-(s / 2)$ and $(1 / 2)+(s / 2)$, respectively. Based on the above analysis, we draw the following observation.

Observation 1. Suppose that the pair $\left(x_{1}^{*}, x_{2}^{*}\right)$ is a Nash equilibrium outcome of players 1 and 2 with the location-price game in the dual-circle market. Then $x_{1}^{*}$ lies in $[(1 / 4),(1 / 2)-$ $(s / 2)]$ and $x_{2}^{*}$ lies in $[(1 / 2)+(s / 2),(1 / 4)]$.

We use the inverse method to explain the rationality of the observation. Assume that $x_{1}^{*} \in[0,(1 / 4))$ and $x_{2}^{*} \in((3 / 4), 1]$. Moving point $x_{1}^{*}$ to $A$ by a small distance $\delta$, player 1 can obtain another position $\tilde{x}_{1}^{*}$, where $\tilde{x}_{1}^{*}=x_{1}^{*}+\sigma$. On the one hand, player 1 at $\tilde{x}_{1}^{*}$ will get more profit from circle $L$ market based on the principle of maximum differentiation for one circle market. On the other hand, since $\tilde{x}_{1}^{*}$ is closer to $A$ than $x_{1}^{*}$ is, it leads to more customers attracted by player 1 . Thus, player 1 at $\tilde{x}_{1}^{*}$ will get more profit from circle $R$ market. Therefore, player 1 at $\tilde{x}_{1}^{*}$ will get more total profit than at $x_{1}^{*}$, which contradicts the fact that the pair $\left(x_{1}^{*}, x_{2}^{*}\right)$ is a Nash equilibrium. So, the pair $\left(x_{1}^{*}, x_{2}^{*}\right)$ cannot be in $[0,1 / 4] \times(3 / 4,1)$. In a similar way, we can explain that neither $x_{1}^{*}$ nor $x_{2}^{*}$ can be in the interval $((1 / 2)-$ $(s / 2),(1 / 2)+(s / 2))$.
Remark 1. Observation 1 suggests, in the location-game, the position pair $\left(x_{1}, x_{2}\right)$ of two players just be considered in the feasible region $[(1 / 4),(1 / 2)-(s / 2)] \times[(1 / 2)+(s / 2),(3 / 4)]$. This consideration is marked different from that in [26], where position $x_{1}$ is fixed at one intersection point $\mathrm{H}_{2}$, while position $x_{2}$ is set in a neighbourhood of another intersection point $H_{1}$. In this paper, since the demand levels of markets $L$ and $R$ may be different, the dual-circle market system is not fully asymmetrical with respect to the axis $\mathrm{H}_{1} \mathrm{H}_{2}$. Consequently, neither $x_{1}$ nor $x_{2}$ should be fixed at point $H_{1}$ or $H_{2}$.

2.3. Attraction Domain of Customers. The net utilities (4) measure the intensity by which a customer at $x$ is willing to buy the products of player $i$. The set of all customers buying the products of player $i$ is called an attraction domain for this player. To determine the boundaries of the attraction domain, we need to examine position $\bar{x}$ of marginal customer whose net utility buying from player 1 or player 2 is indifferent. According to (4), position $\bar{x}$ can be computed from the following equation:

$$
\begin{aligned}
p_{1}+T^{L}\left(d_{\min }\left(\bar{x}, x_{1}\right)\right) & =p_{2}+T^{L}\left(d_{\min }\left(\bar{x}, x_{2}\right)\right), \\
p_{1}+T^{R}\left(d_{\min }\left(\bar{x}, t x_{1}\right)\right) & =p_{2}+T^{R}\left(d_{\min }\left(\bar{x}, x_{2}\right)\right) .
\end{aligned}
$$

For simplicity, we give some notations. Let $L_{i}^{L}$ and $L_{i}^{R}$ be the lengths of attraction domains on circles $L$ and $R$ for player $i$, respectively. Let $m_{1}=(k / 2)\left(x_{2}-x_{1}\right)\left(2+x_{1}-x_{2}\right), m_{2}=$ $(k / 2)\left(x_{2}-x_{1}\right)\left(1-x_{1}+x_{2}\right), \quad m_{3}=(k / 2)\left(1-x_{1}-x_{2}\right)(1-$ $\left.x_{1}+x_{2}\right), n=k s$, and $M=k\left(\left(1 / m_{1}\right)-\left(1 / 2 m_{2}\right)\right)=(3 /(2+$ $\left.\left.x_{1}-x_{2}\right)\left(1-x_{1}+x_{2}\right)\right)$. Obviously, $m_{1} \geq m_{2}>0, \quad m_{2}>$ $m_{3}>-m_{2}, n>0$, and $M>0$. By solving equation (8) to obtain boundaries of the attraction domain of each player, we have the following results on the lengths of attraction domains.

Lemma 1. If customers on circle L choose products neither from player 1 nor from player 2 based on the net utility function (4), positions $\bar{x}_{1}^{L}$ and $\bar{x}_{2}^{L}$ of two indifferent customers as well as lengths $L_{1}^{L}$ and $L_{2}^{L}$ of attraction domains for players 1 and 2 are listed in Table 1 .

The proof of Lemma 1 is presented in the Appendix. 


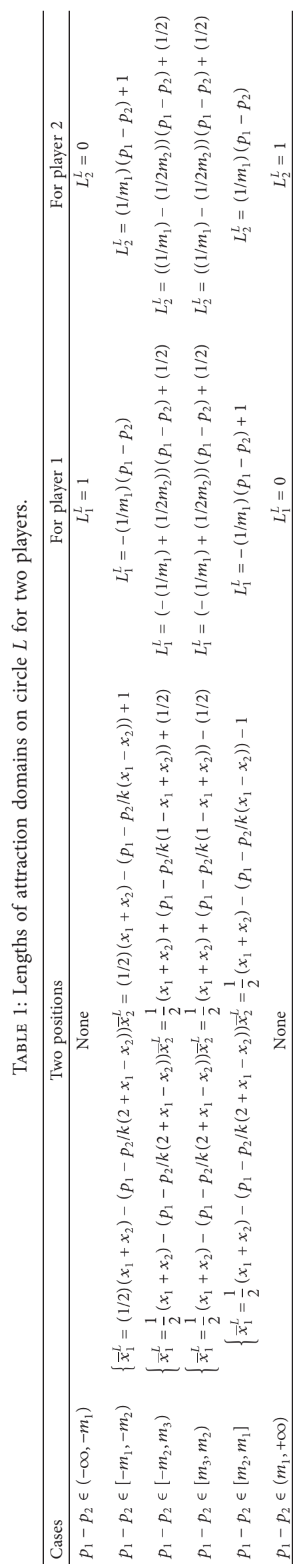


Lemma 2. Let $\Delta T=T^{R}\left(a-x_{1}\right)-T^{R}\left(x_{2}-b\right)=k\left(1-x_{1}\right.$ $\left.-x_{2}\right)$, which is the difference of transportation cost between from $A$ to player 1 and from $B$ to player 2. If customers on circle $R$ choose products neither from player 1 nor from player 2 based on the net utility function (4), lengths $L_{1}^{R}$ and $L_{2}^{R}$ of attraction domains for players 1 and 2 are listed in Table 2.

The proof of Lemma 2 is very similar to that of Lemma 1 , so it is omitted.

Remark 2. From Lemma 1 and Lemma 2, the lengths of attraction domains on circle $R$ and $L$ are both 1 for player 1 if $p_{1}-p_{2}<\min \left\{-m_{1},-n-\Delta T\right\}$, which shows that player 1 occupies the total market by low price.

For the convenience of representation, we make a variable substitution from $\left(x_{1}, x_{2}\right)$ to $(x, y)$ as follows:

$$
\left\{\begin{array}{l}
x=x_{2}-x_{1}, \\
y=x_{1}+x_{2} .
\end{array}\right.
$$

Then the region $\left\{\left(x_{1}, x_{2}\right) \mid(1 / 4) \leq x_{1} \leq(1 / 2)-(s / 2)\right.$, $\left.(1 / 2)+(s / 2) \leq x_{1} \leq(3 / 4)\right\}$ in the $x_{1} O x_{2}$ coordinate system is corresponding to the region $\{(x, y) \mid(1 / 2) \leq y-x \leq 1-s, 1+$ $s \leq x+y \leq(3 / 2)\}$ in the $x O y$ coordinate system, which is the square region $\mathrm{ABCD}$ in Figure 3. In order to express the demand of each player in the form of piecewise function, we need to divide the feasible region properly. As shown in Figure 3, the region ABCD is separated into nine subregions by four curves, where the algebraic expressions of the four curves $l_{1}, l_{2}, l_{3}$, and $l_{4}$ are

$$
\begin{aligned}
& y=\frac{1}{2} x^{2}-x+s+1, \quad x \in[2-2 \sqrt{1-s}, 2-\sqrt{3-2 s}] \\
& y=-\frac{1}{2} x^{2}-\frac{1}{2} x+s+1, \quad x \in\left[-\frac{3}{2}+\frac{1}{2} \sqrt{9+16 s},-\frac{3}{2}+\frac{1}{2} \sqrt{13+8 s}\right], \\
& y=\frac{1}{2} x^{2}+\frac{1}{2} x-s+1, \quad x \in\left[-\frac{3}{2}+\frac{1}{2} \sqrt{9+16 s},-\frac{3}{2}+\frac{1}{2} \sqrt{13+8 s}\right], \\
& y=-\frac{1}{2} x^{2}+x-s+1, \quad x \in[2-2 \sqrt{1-s}, 2-\sqrt{3-2 s}] .
\end{aligned}
$$

For the relationship of end points of intervals of $p_{1}-p_{2}$ in Tables 1 and 2, we have the results on the orders of these end points, which are shown in Table 3 .

Therefore, if two players develop location-price competition in region $I$, the demand function for player 1 can be represented as follows:

$$
D_{1}^{I}\left(p_{1}, p_{2}, x_{1}, x_{2}\right)= \begin{cases}\left(\mu^{L}+\mu^{R}\right) \rho, & p_{1}-p_{2} \in(-\infty,-n-\Delta T), \\ \mu^{L} \rho+\mu^{R} \rho\left[\frac{1}{2}-\frac{1}{k}\left(p_{1}-p_{2}+\Delta T\right)\right], & p_{1}-p_{2} \in\left[-n-\Delta T,-m_{1}\right), \\ -\frac{\mu^{L} \rho}{m_{1}}\left(p_{1}-p_{2}\right)+\mu^{R} \rho\left[\frac{1}{2}-\frac{1}{k}\left(p_{1}-p_{2}+\Delta T\right)\right], & p_{1}-p_{2} \in\left[-m_{1},-m_{2}\right), \\ \mu^{L} \rho\left[\left(-\frac{1}{m_{1}}+\frac{1}{2 m_{2}}\right)\left(p_{1}-p_{2}\right)+\frac{1}{2}\right]+\mu^{R} \rho\left[\frac{1}{2}-\frac{1}{k}\left(p_{1}-p_{2}+\Delta T\right)\right], & p_{1}-p_{2} \in\left[-m_{2}, m_{2}\right), \\ \mu^{L} \rho\left[1-\frac{1}{m_{1}}\left(p_{1}-p_{2}\right)\right]+\mu^{R} \rho\left[\frac{1}{2}-\frac{1}{k}\left(p_{1}-p_{2}+\Delta T\right)\right], & p_{1}-p_{2} \in\left[m_{2}, m_{1}\right], \\ \mu^{R} \rho\left[\frac{1}{2}-\frac{1}{k}\left(p_{1}-p_{2}+\Delta T\right)\right], & p_{1}-p_{2} \in\left(m_{1}, n-\Delta T\right],\end{cases}
$$

Correspondingly, the demand function of player 2 is $D_{2}^{I}\left(p_{1}, p_{2}, x_{1}, x_{2}\right)=\left(\mu^{L}+\mu^{R}\right) \rho-D_{1}^{I}\left(p_{1}, p_{2}, x_{1}, x_{2}\right)$. If two players develop location-price competition in region II, the demand function for player 1 can be represented as follows: 


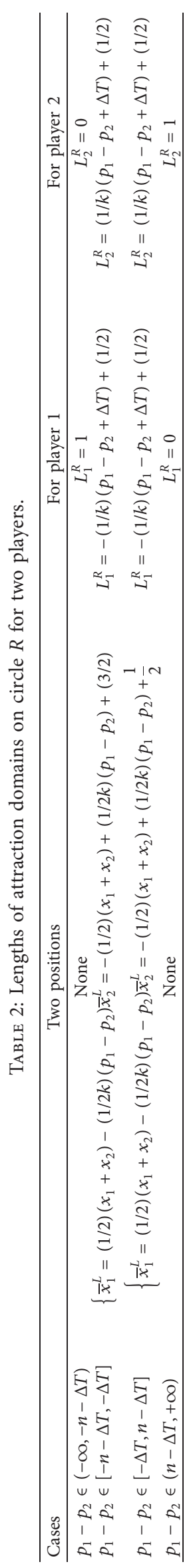




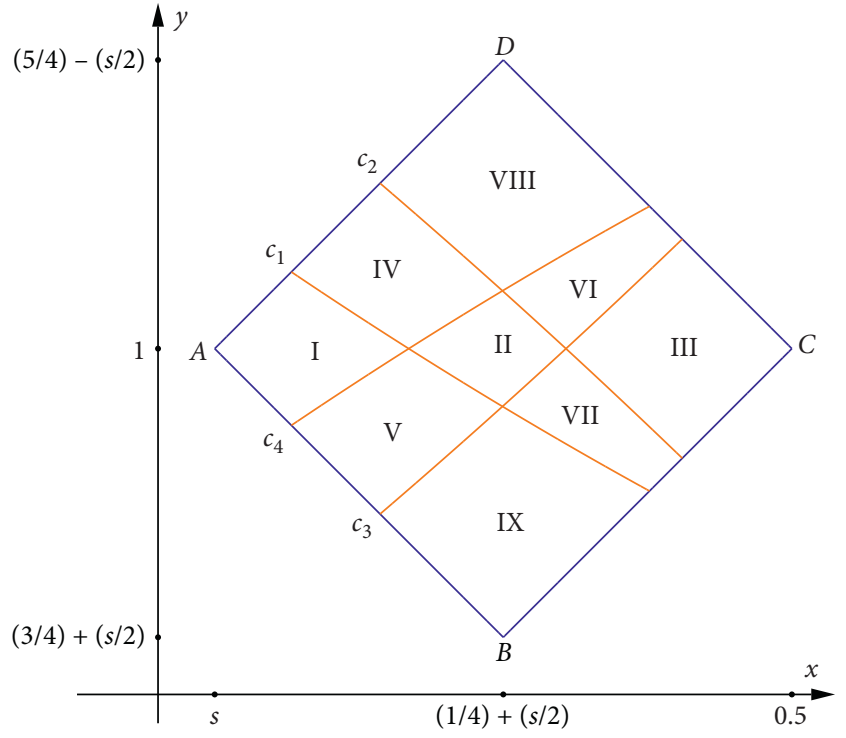

FIgURE 3: Division of the feasible region.
TABLE 3: Orders of end points of intervals of $p_{1}-p_{2}$.

\begin{tabular}{ll}
\hline Cases & \multicolumn{1}{c}{ Orders } \\
\hline$\left(x_{1}, x_{2}\right)$ in region I & $-n-\Delta T \leq-m_{1} \leq-m_{2} \leq m_{2} \leq m_{1} \leq n-\Delta T$ \\
$\left(x_{1}, x_{2}\right)$ in region II & $-m_{1} \leq-n-\Delta T \leq-m_{2} \leq m_{2} \leq n-\Delta T \leq m_{1}$ \\
$\left(x_{1}, x_{2}\right)$ in region & $-m_{1} \leq-m_{2} \leq-n-\Delta T \leq n-\Delta T \leq m_{2} \leq m_{1}$ \\
III & \\
$\left(x_{1}, x_{2}\right)$ in region & $-m_{1} \leq-n-\Delta T \leq-m_{2} \leq m_{2} \leq m_{1} \leq n-\Delta T$ \\
IV & \\
$\left(x_{1}, x_{2}\right)$ in region V & $-n-\Delta T \leq-m_{1} \leq-m_{2} \leq m_{2} \leq n-\Delta T \leq m_{1}$ \\
$\left(x_{1}, x_{2}\right)$ in region & $-m_{1} \leq-m_{2} \leq-n-\Delta T \leq m_{2} \leq n-\Delta T \leq m_{1}$ \\
VI & \\
$\left(x_{1}, x_{2}\right)$ in region & $-m_{1} \leq-n-\Delta T \leq-m_{2} \leq n-\Delta T \leq m_{2} \leq m_{1}$ \\
VII & \\
$\left(x_{1}, x_{2}\right)$ in region & $-m_{1} \leq-m_{2} \leq-n-\Delta T \leq m_{2} \leq m_{1} \leq n-\Delta T$ \\
VIII & \\
$\left(x_{1}, x_{2}\right)$ in region & $-n-\Delta T \leq-m_{1} \leq-m_{2} \leq n-\Delta T \leq m_{2} \leq m_{1}$ \\
IX &
\end{tabular}

$$
D_{1}^{I I}\left(p_{1}, p_{2}, x_{1}, x_{2}\right)= \begin{cases}\left(\mu^{L}+\mu^{R}\right) \rho, & p_{1}-p_{2} \in\left(-\infty,-m_{1}\right), \\ -\frac{\mu^{L} \rho}{m_{1}}\left(p_{1}-p_{2}\right)+\mu^{R} \rho, & p_{1}-p_{2} \in\left[-m_{1},-n-\Delta T\right), \\ -\frac{\mu^{L} \rho}{m_{1}}\left(p_{1}-p_{2}\right)+\mu^{R} \rho\left[\frac{1}{2}-\frac{1}{k}\left(p_{1}-p_{2}+\Delta T\right)\right], & p_{1}-p_{2} \in\left[-n-\Delta T,-m_{2}\right), \\ \mu^{L} \rho\left[\left(-\frac{1}{m_{1}}+\frac{1}{2 m_{2}}\right)\left(p_{1}-p_{2}\right)+\frac{1}{2}\right]+\mu^{R} \rho\left[\frac{1}{2}-\frac{1}{k}\left(p_{1}-p_{2}+\Delta T\right)\right], & p_{1}-p_{2} \in\left[-m_{2}, m_{2}\right), \\ \mu^{L} \rho\left[1-\frac{1}{m_{1}}\left(p_{1}-p_{2}\right)\right]+\mu^{R} \rho\left[\frac{1}{2}-\frac{1}{k}\left(p_{1}-p_{2}+\Delta T\right)\right], & p_{1}-p_{2} \in\left[m_{2}, n-\Delta T\right], \\ \mu^{L} \rho\left[1-\frac{1}{m_{1}}\left(p_{1}-p_{2}\right)\right], & p_{1}-p_{2} \in\left(n-\Delta T, m_{1}\right],\end{cases}
$$

Then the demand function of player 2 is $D_{2}^{I I}\left(p_{1}, p_{2}, x_{1}, x_{2}\right)=\left(\mu^{L}+\mu^{R}\right) \rho-D_{1}^{I I}\left(p_{1}, p_{2}, x_{1}, x_{2}\right)$. If two players develop location-price competition in region III, the demand function for player 1 can be represented as follows: 


$$
D_{1}^{I I I}\left(p_{1}, p_{2}, x_{1}, x_{2}\right)= \begin{cases}\left(\mu^{L}+\mu^{R}\right) \rho, & p_{1}-p_{2} \in\left(-\infty,-m_{1}\right), \\ -\frac{\mu^{L} \rho}{m_{1}}\left(p_{1}-p_{2}\right)+\mu^{R} \rho, & p_{1}-p_{2} \in\left[-m_{1},-m_{2}\right), \\ \mu^{L} \rho\left[\left(-\frac{1}{m_{1}}+\frac{1}{2 m_{2}}\right)\left(p_{1}-p_{2}\right)+\frac{1}{2}\right]+\mu^{R} \rho, & p_{1}-p_{2} \in\left[-m_{2},-n-\Delta T\right), \\ \mu^{L} \rho\left[\left(-\frac{1}{m_{1}}+\frac{1}{2 m_{2}}\right)\left(p_{1}-p_{2}\right)+\frac{1}{2}\right]+\mu^{R} \rho\left[\frac{1}{2}-\frac{1}{k}\left(p_{1}-p_{2}+\Delta T\right)\right], & p_{1}-p_{2} \in[-n-\Delta T, n-\Delta T], \\ \mu^{L} \rho\left[\left(-\frac{1}{m_{1}}+\frac{1}{2 m_{2}}\right)\left(p_{1}-p_{2}\right)+\frac{1}{2}\right], & p_{1}-p_{2} \in\left(n-\Delta T, m_{2}\right), \\ \mu^{L} \rho\left[1-\frac{1}{m_{1}}\left(p_{1}-p_{2}\right)\right], & p_{1}-p_{2} \in\left[m_{2}, m_{1}\right], \\ 0, & p_{1}-p_{2} \in\left(m_{1},+\infty\right) .\end{cases}
$$

In addition, the demand function of player 2 is $D_{2}^{I I I}\left(p_{1}, p_{2}, x_{1}, x_{2}\right)=\left(\mu^{L}+\mu^{R}\right) \rho-D_{1}^{I I I}\left(p_{1}, p_{2}, x_{1}, x_{2}\right)$. For saving space, we just list the demand functions in regions I, II, and III in this paper.

\section{Location-Price Game}

In this section, we consider the location game as a two-stage pattern. In the first stage of the game, the two firms choose their locations $x_{1}$ and $x_{2}$ independently. In the second stage, the firms determine their prices $p_{1}$ and $p_{2}$ after observing the locations. We employ backward induction method to solve the game.

In the second stage, firms simultaneously determine their prices at each position in region $\mathrm{ABCD}$. In the following, we only discuss the case of the positions in region I. For other cases, the discussion is very similar. Since the positions of two players are in region I, their profits are

$$
\pi_{i}\left(p_{1}, p_{2}, x_{1}, x_{2}\right)=p_{i} D_{i}^{I}\left(p_{1}, p_{2}, x_{1}, x_{2}\right),
$$

where $i=1,2$. To obtain a price equilibrium, we need to solve the following equations derived from the first-order conditions of the profits in $p_{1}$ and $p_{2}$ :

$$
\begin{aligned}
& \frac{\partial \pi_{1}}{\partial p_{1}}=0, \\
& \frac{\partial \pi_{2}}{\partial p_{2}}=0 .
\end{aligned}
$$

We discuss the solutions of equations (15) in seven cases: (i) $p_{1}-p_{2} \in(-\infty,-n-\Delta T)$; (ii) $p_{1}-p_{2} \in\left[-n-\Delta T,-m_{1}\right)$; (iii) $p_{1}-p_{2} \in\left[-m_{1},-m_{2}\right)$; (iv) $p_{1}-p_{2} \in\left[-m_{2}, m_{2}\right)$; (v) $p_{1}-p_{2} \in\left[m_{2}, m_{1}\right] ;$ (vi) $p_{1}-p_{2} \in\left(m_{1}, n-\Delta T\right]$; and (vii) $p_{1}-p_{2} \in(n-\Delta T,+\infty)$. It will be shown that equation (15) has a solution in case (iv), while the equations have no solutions in other cases.

Obviously, there is no solution in equation (15) for cases (i) and (vii).

For case (ii), equation (15) is equivalent to

$$
\left\{\begin{array}{l}
-\frac{2 \mu^{R}}{k} p_{1}+\frac{\mu^{R}}{k} p_{2}+\mu^{L}+\mu^{R}\left(\frac{1}{2}-\frac{\Delta T}{k}\right)=0, \\
\frac{2 \mu^{R}}{k} p_{1}+\frac{2 \mu^{R}}{k} p_{2}+\mu^{R}\left(\frac{1}{2}+\frac{\Delta T}{k}\right)=0 .
\end{array}\right.
$$

Subtracting the two equations in (16), we have that

$$
p_{1}-p_{2}=\frac{1}{3}\left(\frac{k \mu^{L}}{\mu^{R}}-2 \Delta T\right) \text {. }
$$

However, $p_{1}-p_{2}$ in (17) does not lie in $\left[-n-\Delta T,-m_{1}\right)$, since $-(2 / 3) \Delta T>-m_{1}$. Therefore, equation (15) has no solutions for case (ii).

For case (iii), equation (15) is equivalent

$$
\left\{\begin{array}{l}
-\left(\frac{\mu^{L}}{m_{1}}+\frac{\mu^{R}}{k}\right)\left(2 p_{1}-p_{2}\right)+\mu^{R}\left(\frac{1}{2}-\frac{\Delta T}{k}\right)=0, \\
\left(\frac{\mu^{L}}{m_{1}}+\frac{\mu^{R}}{k}\right)\left(p_{1}-2 p_{2}\right)+\mu^{R}\left(\frac{1}{2}+\frac{\Delta T}{k}\right)+\mu^{L}=0 .
\end{array}\right.
$$

Subtracting the two equations in (18), we have that 


$$
3\left(\frac{\mu^{L}}{m_{1}}+\frac{\mu^{R}}{k}\right)\left(p_{1}-p_{2}\right)+\frac{2 \mu^{R} \Delta T}{k}+\mu^{L}=0 .
$$

Therefore, $p_{1}$ and $p_{2}$ should satisfy condition (19) if $\left(p_{1}, p_{2}\right)$ is a solution of (18). However, condition (19) does not hold for any $\left(p_{1}, p_{2}\right)$ in case (iii). The reason is that

$$
\begin{aligned}
& 3\left(\frac{\mu^{L}}{m_{1}}+\frac{\mu^{R}}{k}\right)\left(p_{1}-p_{2}\right)+\frac{2 \mu^{R} \Delta T}{k} \\
& \quad+\mu^{L} \leq-3 m_{2}\left(\frac{\mu^{L}}{m_{1}}+\frac{\mu^{R}}{k}\right)+\frac{2 \mu^{R} \Delta T}{k}+\mu^{L} \\
& =\mu^{L}\left[1-\frac{3\left(1+X_{1}\right)}{2-X_{1}}\right]+\mu^{R}\left[-\frac{3}{2} X_{1}\left(1+X_{1}\right)+2\left(1-X_{2}\right)\right] \\
& \leq-\mu^{L}\left(\frac{4 s+1}{2-s}\right)-\mu^{R}\left(\frac{3}{2} s^{2}+\frac{3}{2} s\right)<0 .
\end{aligned}
$$

Therefore, equation (15) has no solutions for case (iii).

For cases (v) and (vi), there are no solutions in equation (15). The proofs are very similar to those of cases (ii) and (iii), so they are omitted.

For case (iv), equation (15) is equivalent to

$$
\left\{\begin{array}{l}
-\frac{1}{k}\left(\mu^{L} M+\mu^{R}\right)\left(2 p_{1}-p_{2}\right)-\mu^{R}(1-y)+\frac{1}{2}\left(\mu^{L}+\mu^{R}\right)=0, \\
\frac{1}{k}\left(\mu^{L} M+\mu^{R}\right)\left(p_{1}-2 p_{2}\right)+\mu^{R}(1-y)+\frac{1}{2}\left(\mu^{L}+\mu^{R}\right)=0 .
\end{array}\right.
$$

Solving the equation, we can obtain $p_{1}^{*}=((2 y+3 \theta+1)$ $k / 6(\theta M(x)+1))$ and $p_{2}^{*}=((-2 y+3 \theta+5) k / 6(\theta M(x)+$ $1))$. Furthermore, it can be calculated that

$$
\left|p_{1}^{*}-p_{2}^{*}\right|=\frac{2|y-1| k}{3(\theta M(x)+1)} \leq \frac{2 k}{3}|y-1|<m_{2} \text {. }
$$

Therefore, $-n-\Delta T \leq-m_{2}<p_{1}^{*}-p_{2}^{*}<m_{2} \leq n-\Delta T$.

Summarizing the above discussion, we obtain the proposition on the price equilibrium.

Proposition 1. In the location-price game in the dual-circle market, there exists a price equilibrium $\left(p_{1}^{*}, p_{2}^{*}\right)$ for any $\left(x_{1}, x_{2}\right)$ located in region $A B C D$, which can be determined by

$$
\begin{aligned}
& p_{1}^{*}\left(x_{1}, x_{2}\right)=\frac{(2 y+3 \theta+1) k}{6(\theta M(x)+1)}, \\
& p_{2}^{*}\left(x_{1}, x_{2}\right)=\frac{(-2 y+3 \theta+5) k}{6(\theta M(x)+1)},
\end{aligned}
$$

where $\theta=\left(\mu^{L} / \mu^{R}\right)$ and $M(x)=(3 /(2-x)(1+x))$. Moreover,

$$
-\min \left\{m_{2}, n+\Delta T\right\} \leq p_{1}^{*}-p_{2}^{*} \leq \min \left\{m_{2}, n-\Delta T\right\} .
$$

Remark 3. From (25), it is shown that prices $p_{1}$ and $p_{2}$ of two players are not very different, when the price game is in equilibrium. Note that

$$
\begin{gathered}
\left|\begin{array}{cc}
3 & 2 y+1 \\
M(x) & 1
\end{array}\right|<0, \\
\left|\begin{array}{cc}
3 & -2 y+5 \\
M(x) & 1
\end{array}\right|<0 .
\end{gathered}
$$

It follows from (23) and (24) that $\left(\mathrm{d} p_{1}^{*} / \mathrm{d} \theta\right)<0$ and $\left(\mathrm{d} p_{2}^{*} / \mathrm{d} \theta\right)<0$. Therefore, $p_{1}^{*}$ and $p_{2}^{*}$ are monotonically decreasing with respect to $\theta$. It is revealed that if the increase of domestic demand level is greater than that of international demand level, the price competition between two players will be intensified, which may lead to an undercutting phenomenon.

Back to the first stage, each firm chooses an optimal location such that its profit cannot be Pareto improved. Before we discuss this, let us propose the following lemma ensuring that the optimal locations can be calculated directly.

Lemma 3. Consider $s \in((1 / 6),(1 / 2))$ and

$$
\theta_{0}=\frac{-B+\sqrt{B^{2}-4 A C}}{2 A},
$$

where $A=9(1-2 s), B=9(1-2 s)-12(2-s)(1+s)$, and $C=-4(2-s)^{2}(1+s)^{2}$. Let $G(x, \theta)=12 \theta(2-x)(1+x)+4$ $(2-x)^{2}(1+x)^{2}-9 \theta(\theta+1)(1-2 x)$. If $\theta \geq \theta_{0}$, the following equation of $x$,

$$
G(x, \theta)=0
$$

has a unique solution $x^{*}$ in $[s,(1 / 2)]$, which can be given by

$$
x^{*}(\theta)=\frac{1}{2}-\frac{\xi-\sqrt{\xi^{2}-4(h-\zeta)}}{2},
$$

where $h=a+\eta, \quad \xi=\sqrt{2 \eta+a}, \quad \zeta=-(b / 2 \xi), \quad \eta=\sqrt{[3]}-$ $(\beta / 2)+\sqrt{\delta}+\sqrt{[3]}-(\beta / 2)-\sqrt{\delta}-(5 / 6) a, \quad \delta=\left(\alpha^{3} / 27\right)+$ $\left(\beta^{2} / 4\right), \alpha=-\left(a^{2} / 12\right)-c, \beta=-\left(a^{3} / 108\right)+(a c / 3)-\left(b^{2} / 8\right)$, $a=-(9 / 2)-3 \theta, \quad b=-(9 / 2) \theta(\theta+1), \quad$ and $c=(27 / 4) \theta+$ $(81 / 16)$. Furthermore, $\left(d x^{*} / d \theta\right)>0$ for all $\theta \in\left[\theta_{0},+\infty\right)$.

The proof of Lemma 3 is presented in the Appendix.

In the location subgame, the profit functions of players 1 and 2 are written as 


$$
\left\{\begin{array}{l}
\pi_{1}=p_{1}^{*}\left\{\mu^{L} \rho\left[\left(-\frac{1}{m_{1}}+\frac{1}{2 m_{2}}\right)\left(p_{1}^{*}-p_{2}^{*}\right)+\frac{1}{2}\right]+\mu^{R} \rho\left[\frac{1}{2}-\frac{1}{k}\left(p_{1}^{*}-p_{2}^{*}+\Delta T\right)\right]\right\}, \\
\pi_{2}=p_{2}^{*}\left\{\left(\mu^{L}+\mu^{R}\right) \rho-\mu^{L} \rho\left[\left(-\frac{1}{m_{1}}+\frac{1}{2 m_{2}}\right)\left(p_{1}^{*}-p_{2}^{*}\right)-\frac{1}{2}\right]+\mu^{R} \rho\left[\frac{1}{2}-\frac{1}{k}\left(p_{1}^{*}-p_{2}^{*}+\Delta T\right)\right]\right\},
\end{array}\right.
$$

for any $\left(x_{1}, x_{2}\right)$ in region $\mathrm{ABCD}$, where $p_{1}^{*}$ and $p_{2}^{*}$ satisfy condition (25) by Proposition 1 . The equations can be reduced to

$$
\left\{\begin{array}{l}
\pi_{1}=\frac{k \mu^{R} \rho(2 y+3 \theta+1)^{2}}{36(\theta M(x)+1)} \\
\pi_{2}=\frac{k \mu^{R} \rho(-2 y+3 \theta+5)^{2}}{36(\theta M(x)+1)}
\end{array}\right.
$$

where $\theta$ and $M(x)$ are defined in Proposition 1. According to the following first-order conditions of the profits in $x_{1}$ and $x_{2}$,

$$
\begin{aligned}
& \frac{\partial \pi_{1}}{\partial x_{1}}=0, \\
& \frac{\partial \pi_{2}}{\partial x_{2}}=0, \\
& \frac{-b \pm \sqrt{b^{2}-4 a c}}{2 a} .
\end{aligned}
$$

We see that

$$
\left\{\begin{array}{l}
4(\theta M(x)+1)-\frac{3 \theta(2 y+3 \theta+1)(1-2 x)}{(2-x)^{2}(1+x)^{2}}=0, \\
-4(\theta M(x)+1)+\frac{3 \theta(-2 y+3 \theta+5)(1-2 x)}{(2-x)^{2}(1+x)^{2}}=0 .
\end{array}\right.
$$

Adding and subtracting the above two equations, the equivalent equations are obtained as follows:

$$
\left\{\begin{array}{l}
G(x, \theta)=0, \\
y=1,
\end{array}\right.
$$

where $G(x, \theta)$ is defined in (28) in Lemma 3. Therefore, $x^{*}$ defined in (29) and $y^{*}=1$ are a solution pair of (34), which are equivalent to (32). Then the solution of (32) is $x_{1}^{*}=$ $(1 / 2)-(1 / 2) x^{*}$ and $x_{2}^{*}=(1 / 2)+(1 / 2) x^{*}$ because of $(9)$.

Based on the above discussion, we summarize what has been proved.

Proposition 2. In the location-price game in the dual-circle market, the two players choose the price policy of (23) and (24) based on their positions $\left(x_{1}, x_{2}\right)$ located in region $A B C D$. If the proportion factor $\theta=\left(\rho^{L} / \rho^{R}\right) \geq \theta_{0}$, there exists a location equilibrium $\left(x_{1}^{*}, x_{2}^{*}\right)$, which can be determined by

$$
\begin{aligned}
& x_{1}^{*}=\frac{1}{2}-\frac{1}{2} x^{*}, \\
& x_{2}^{*}=\frac{1}{2}+\frac{1}{2} x^{*},
\end{aligned}
$$

where $\theta_{0}$ and $x^{*}$ are determined by (27) and (29) in Lemma 3.

Remark 4. Proposition 2 indicates that the location equilibrium $\left(x_{1}^{*}, x_{2}^{*}\right)$ exists and lies in $[(1 / 4),(1 / 2)-(s / 2)] \times$ $[(1 / 2)+(s / 2),(3 / 4)]$ if $\theta \geq \theta_{0}$. In fact, if $\theta<\theta_{0}$, the location equilibrium will be at positions $H_{1}$ and $H_{2}$ of two hubs; that is, $\left(x_{1}^{*}, x_{2}^{*}\right)=((1 / 2)-(s / 2),(1 / 2)+(s / 2))$, according to the explanation of Observation 1 . Noting that $\left(\mathrm{d} x^{*} / \mathrm{d} \theta\right)>0$, we see that

$$
\begin{aligned}
& \lim _{\theta \longrightarrow \theta_{0}+} x_{1}^{*}=\frac{1}{2}-\frac{s}{2}, \\
& \lim _{\theta \longrightarrow \theta_{0}+} x_{2}^{*}=\frac{1}{2}+\frac{s}{2}, \\
& \lim _{\theta \longrightarrow+\infty} x_{1}^{*}=\frac{1}{4}, \\
& \lim _{\theta \longrightarrow+\infty} x_{2}^{*}=\frac{3}{4},
\end{aligned}
$$

which means that $x_{1}^{*}$ and $x_{2}^{*}$ will be close to (1/4) and (3/4), respectively, if the level of domestic demand is much higher than that of international demand. Otherwise, $x_{1}^{*}$ and $x_{2}^{*}$ will be close to the hubs' positions when $\theta$ approaches $\theta_{0}$.

\section{Numerical Simulations}

To illustrate the competitive dynamics in the dual-circle market and verify the validity of the results, we design an algorithm to simulate the game process of players 1 and 2, which is presented in Algorithm 1. In the simulation, two players move around circle $L$ round by round after they randomly and independently choose points $x_{1}$ and $x_{2}$ as their initial positions. In each round, player 1 first adjusts the position based on the rival's location to obtain a maximum profit. Then, player 2 adjusts the position using the same method as player 1 does. In this way, the two players move alternately on the circle until neither of their profits increase.

Let $s=(1 / 4), k=1, \rho=1, \mu^{L}=4$, and $\mu^{R}=1$. Then $\theta=\left(\mu^{L} / \mu^{R}\right)=4$. On the one hand, we can compute that the location equilibrium is $x_{1}^{*}=0.4179$ and $x_{2}^{*}=0.5821$ according to (35). On the other hand, the final positions of the two players are $x_{1}=0.4179$ and $x_{2}=0.5822$ after 100 rounds of adjustment by Algorithm 1, which are almost the same as $x_{1}^{*}$ and $x_{2}^{*}$ computed by (35). Figures 4 and 5 show 
(1) Initialize parameters $k, \rho, s, \mu^{L}$, and $\mu^{R}$ and compute $\theta=\left(\mu^{L} / \mu^{R}\right)$.

(2) Initialize $x_{1}$ and $x_{2}$ with two random numbers in [(1/4), (1/2)-(s/2)] and [(1/2)+(s/2), (3/4), ], respectively.

(3) Set New $x_{1}=0, \mathrm{New} x_{2}=0$, and $h=0.000001$.

(4) Compute $p_{1}=p_{1}^{*}\left(x_{1}, x_{2}\right)$ and $p_{2}=p_{2}^{*}\left(x_{1}, x_{2}\right)$ according to (23) and (24).

(5) Compute $\pi_{1}$ and $\pi_{2}$ according to (30).

(6) while $\mathrm{New} x_{1} \neq x_{1}$ or New $x_{2} \neq x_{2}$ do

(7) while New $x_{1} \neq x_{1}$ do

(8) $\quad x_{1}=\operatorname{New} x_{1}, \pi_{1}=\operatorname{New} \pi_{1}$.

(9) Compute $p_{1}=p_{1}^{*}\left(x_{1}+h, x_{2}\right)$ and $p_{2}=p_{2}^{*}\left(x_{1}+h, x_{2}\right)$ according to (23) and (24).

(10) Compute New $\pi_{11}=\pi_{1}\left(p_{1}, p_{2}, x_{1}+h, x_{2}\right)$ according to (30).

(11) Compute $p_{1}=p_{1}^{*}\left(x_{1}-h, x_{2}\right)$ and $p_{2}=p_{2}^{*}\left(x_{1}-h, x_{2}\right)$ according to (23) and (24).

(12) Compute New $\pi_{12}=\pi_{1}\left(p_{1}, p_{2}, x_{1}-h, x_{2}\right)$ according to (30).

(13) if New $\pi_{11}>\pi_{1}$ then

(14) $\quad \operatorname{New} x_{1}=x_{1}+h, \operatorname{New} \pi_{1}=\operatorname{New} \pi_{11}$.

(15) end if

(16) if New $\pi_{12}>\pi_{1}$ then

(17) $\quad \operatorname{New} x_{1}=x_{1}-h, \operatorname{New} \pi_{1}=\operatorname{New} \pi_{12}$.

(18) end if

(19) end while

(20) while $\mathrm{New} x_{2} \neq x_{2}$ do

(21) $\quad x_{2}=\operatorname{New} x_{2}, \pi_{2}=N e w \pi_{2}$.

(22) Compute $p_{1}=p_{1}^{*}\left(x_{1}, x_{2}+h\right)$ and $p_{2}=p_{2}^{*}\left(x_{1}, x_{2}+h\right)$ according to (23) and (24).

(23) Compute New $\pi_{21}=\pi_{2}\left(p_{1}, p_{2}, x_{1}, x_{2}+h\right)$ according to (30).

(24) Compute $p_{1}=p_{1}^{*}\left(x_{1}, x_{2}-h\right)$ and $p_{2}=p_{2}^{*}\left(x_{1}, x_{2}-h\right)$ according to (23) and (24).

(25) Compute New $\pi_{22}=\pi_{2}\left(p_{1}, p_{2}, x_{1}, x_{2}-h\right)$ according to (30).

(26) if New $\pi_{21}>\pi_{2}$ then

(27) $\quad \operatorname{New} x_{2}=x_{2}+h, \operatorname{New} \pi_{2}=\operatorname{New} \pi_{21}$.

(28) end if

(29) if New $\pi_{22}>\pi_{2}$ then

(30) $\quad \mathrm{New} x_{2}=x_{2}-h, \mathrm{New} \pi_{2}=\mathrm{New} \pi_{22}$.

(31) end if

(32) end while

(33) end while

(34) return New $x_{1}$ and New $x_{2}$.

Algorithm 1: Location-price game algorithm.

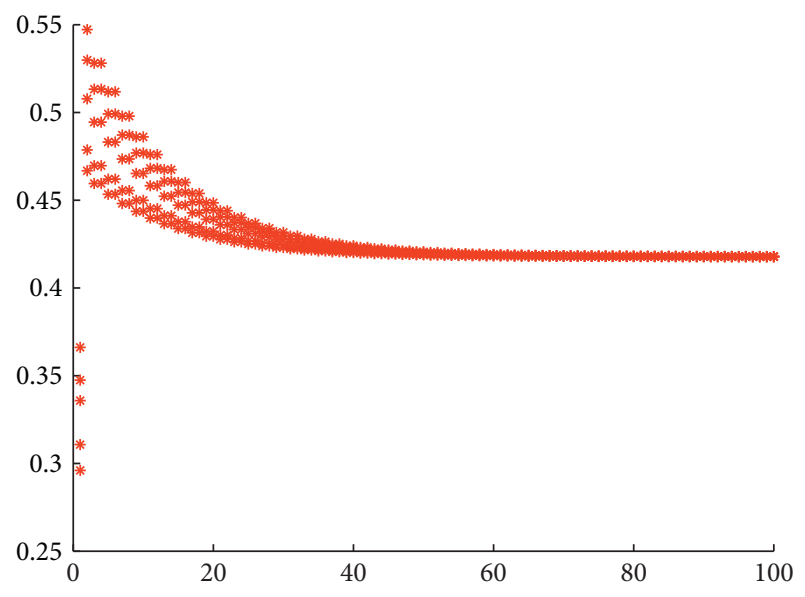

Figure 4: Player 1's dynamic evolution of 100 rounds with 5 random initial positions.

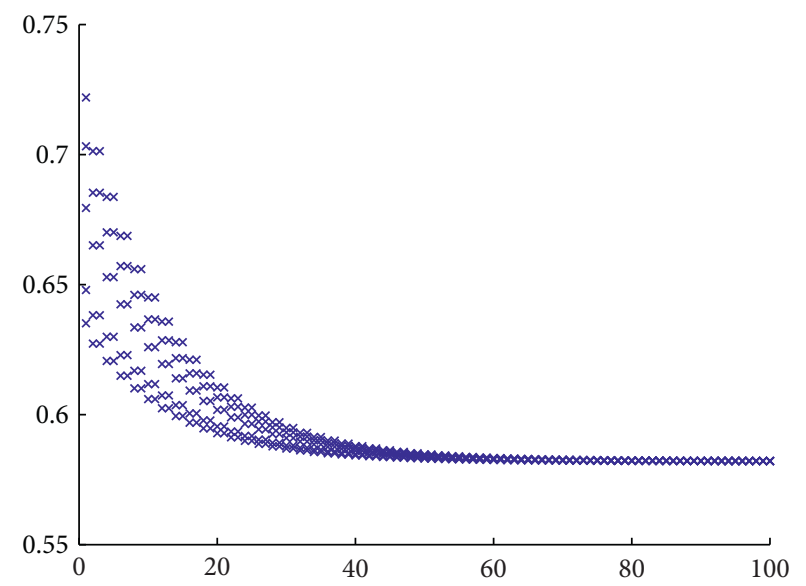

Figure 5: Player 2's dynamic evolution of 100 rounds with 5 random initial positions. 
two players' dynamic evolution of 100 rounds with 5 random initial positions, from which it is seen that two players almost reach the equilibrium positions $x_{1}^{*}$ and $x_{2}^{*}$ obtained by theory, wherever their initial positions are.

\section{Conclusion}

To study the location-price game in a dual-circle market system including domestic and international markets with different demand levels, we consider the shape of the market as two intersecting circles. Employing a two-stage method, we solve the subgame-perfect Nash equilibria of the location game in the dual-circle market. In addition, we design an algorithm to simulate the dynamic evolution of the locationprice game in the dual-circle market and make a numerical simulation to illustrate and validate the obtained results. Due to the impact of the recent COVID-19 pandemic, the domestic and international market demand levels have decreased to varying degrees. Therefore, it is practically significant to consider the location-price game with the different demand levels in two markets in this paper. The obtained results show that firms would like to look for their new equilibrium locations when the domestic demand level is larger than the international one to a certain extent. Otherwise, they should be in their original equilibrium positions that are near the transportation hubs. This paper considers that customers are evenly distributed over each circle market with a constant density. In fact, the density of customers may not be constant in the real world. Therefore, we will mainly focus on the research of the location game with uneven customer distributions.

\section{Appendix}

The proof of Lemma 1. If $p_{1}-p_{2} \in\left(-\infty,-m_{1}\right)$, there is no solution of $(8)$, since $p_{1}+T^{L}\left(d_{\min }\left(\bar{x}, x_{1}\right)\right)<p_{2}+T^{L}$ $\left(d_{\min }\left(\bar{x}, x_{2}\right)\right)$. Therefore, $R_{1}^{L}=1$ and $R_{2}^{L}=0$.

If $p_{1}-p_{2} \in\left(m_{1},+\infty\right)$, there is no solution of (8), since $p_{1}+c\left(d_{\text {min }}\left(\bar{x}, x_{1}\right)\right)>p_{2}+c\left(d_{\min }\left(\bar{x}, x_{2}\right)\right)$. Therefore, $R_{1}^{L}=0$ and $R_{2}^{L}=1$.

If $p_{1}-p_{2} \in\left[-m_{1}, m_{1}\right]$, there exists an indifferent customer in interval $\left[x_{1}, x_{2}\right]$. According to (3) and (6), equation (8) can be changed into

$$
\begin{aligned}
p_{1}-p_{2}= & k\left[\left(x_{2}-\bar{x}_{1}^{L}\right)-\frac{1}{2}\left(x_{2}-\bar{x}_{1}^{L}\right)^{2}\right] \\
& -k\left[\left(\bar{x}_{1}^{L}-x_{1}\right)-\frac{1}{2}\left(\bar{x}_{1}^{L}-x_{1}\right)^{2}\right] .
\end{aligned}
$$

Solving the equation above, we have

$$
\bar{x}_{1}^{L}=\frac{1}{2}\left(x_{1}+x_{2}\right)-\frac{p_{1}-p_{2}}{k\left(2+x_{1}-x_{2}\right)} .
$$

If $p_{1}-p_{2} \in\left[-m_{1}, m_{1}\right]$, there also exists another indifferent customer in intervals $\left[x_{2}, 1\right) \cup\left[0, x_{1}\right]$. For this situation, we consider position $\bar{x}_{2}^{L}$ of the indifferent customer in four cases: (i) $p_{1}-p_{2} \in\left[-m_{1},-m_{2}\right)$, (ii) $p_{1}-p_{2} \in\left[-m_{2}, m_{3}\right.$ ), (iii) $p_{1}-p_{2} \in\left[m_{3}, m_{2}\right)$, and (iv) $p_{1}-p_{2} \in\left[m_{2}, m_{1}\right]$.
In case (i), where $p_{1}-p_{2} \in\left[-m_{1},-m_{2}\right)$, position $\bar{x}_{2}^{L}$ lies in $\left[x_{2}, x_{1}+(1 / 2)\right)$. According to (3) and (6), equation (8) can be changed into

$$
\begin{aligned}
p_{1}-p_{2}= & k\left[\left(\bar{x}_{2}^{L}-x_{2}\right)-\frac{1}{2}\left(\bar{x}_{2}^{L}-x_{2}\right)^{2}\right] \\
& -k\left[\left(\bar{x}_{2}^{L}-x_{1}\right)-\frac{1}{2}\left(\bar{x}_{2}^{L}-x_{1}\right)^{2}\right] .
\end{aligned}
$$

Solving the equation above, we have

$$
\bar{x}_{2}^{L}=\frac{1}{2}\left(x_{1}+x_{2}\right)-\frac{p_{1}-p_{2}}{k\left(x_{1}-x_{2}\right)}+1
$$

Then the lengths of attraction domains are

$$
\begin{aligned}
L_{2}^{L} & =\bar{x}_{2}^{L}-\bar{x}_{1}^{L}=1-\frac{p_{1}-p_{2}}{k\left(x_{1}-x_{2}\right)}+\frac{p_{1}-p_{2}}{k\left(2+x_{1}-x_{2}\right)} \\
& =1-\frac{2\left(p_{1}-p_{2}\right)}{k\left(x_{1}-x_{2}\right)\left(2+x_{1}-x_{2}\right)}=\frac{1}{m_{1}}\left(p_{1}-p_{2}\right)+1, \\
L_{1}^{L} & =1-L_{2}^{L}=-\frac{1}{m_{1}}\left(p_{1}-p_{2}\right) .
\end{aligned}
$$

In case (ii), where $p_{1}-p_{2} \in\left[-m_{2}, m_{3}\right)$, position $\bar{x}_{2}^{L}$ lies in $\left[x_{1}+(1 / 2), 1\right]$. According to (3) and (6), equation (8) can be changed into

$$
\begin{aligned}
p_{1}-p_{2}= & k\left[\left(\bar{x}_{2}^{L}-x_{2}\right)-\frac{1}{2}\left(\bar{x}_{2}^{L}-x_{2}\right)^{2}\right] \\
& -k\left[\left(1-\bar{x}_{2}^{L}+x_{1}\right)-\frac{1}{2}\left(1-\bar{x}_{2}^{L}+x_{1}\right)^{2}\right] .
\end{aligned}
$$

Solving the equation above, we have

$$
\bar{x}_{2}^{L}=\frac{1}{2}\left(x_{1}+x_{2}\right)+\frac{p_{1}-p_{2}}{k\left(1-x_{1}+x_{2}\right)}+\frac{1}{2} .
$$

Then, the lengths of attraction domains are

$$
\begin{aligned}
L_{2}^{L} & =\bar{x}_{2}^{L}-\bar{x}_{1}^{L}=\frac{1}{2}+\frac{p_{1}-p_{2}}{k\left(1-x_{1}+x_{2}\right)}+\frac{p_{1}-p_{2}}{k\left(2+x_{1}-x_{2}\right)} \\
& =\frac{1}{2}+\frac{3\left(p_{1}-p_{2}\right)}{k\left(1-x_{1}+x_{2}\right)\left(2+x_{1}-x_{2}\right)}=\left(\frac{1}{m_{1}}-\frac{1}{2 m_{2}}\right)\left(p_{1}-p_{2}\right)+\frac{1}{2}, \\
L_{1}^{L} & =1-L_{2}^{L}=\left(-\frac{1}{m_{1}}+\frac{1}{2 m_{2}}\right)\left(p_{1}-p_{2}\right)+\frac{1}{2} .
\end{aligned}
$$

In case (iii), where $p_{1}-p_{2} \in\left[m_{3}, m_{2}\right)$, position $\bar{x}_{2}^{L}$ lies in $\left[0, x_{2}-(1 / 2)\right)$. According to (3) and (6), equation (8) can be changed into

$$
\begin{aligned}
p_{1}-p_{2}= & k\left[\left(1-x_{2}+\bar{x}_{2}^{L}\right)-\frac{1}{2}\left(1-x_{2}+\bar{x}_{2}^{L}\right)^{2}\right] \\
& -k\left[\left(x_{1}-\bar{x}_{2}^{L}\right)-\frac{1}{2}\left(x_{1}-\bar{x}_{2}^{L}\right)^{2}\right] .
\end{aligned}
$$

Solving the equation above, we have 


$$
\bar{x}_{2}^{L}=\frac{1}{2}\left(x_{1}+x_{2}\right)+\frac{p_{1}-p_{2}}{k\left(1-x_{1}+x_{2}\right)}-\frac{1}{2} .
$$

Then, the lengths of attraction domains are

$$
\begin{aligned}
L_{1}^{L} & =\bar{x}_{1}^{L}-\bar{x}_{2}^{L}=\frac{1}{2}-\frac{p_{1}-p_{2}}{k\left(2+x_{1}-x_{2}\right)}-\frac{p_{1}-p_{2}}{k\left(1-x_{1}+x_{2}\right)} \\
& =\frac{1}{2}-\frac{3\left(p_{1}-p_{2}\right)}{k\left(1-x_{1}+x_{2}\right)\left(2+x_{1}-x_{2}\right)}=\left(-\frac{1}{m_{1}}+\frac{1}{2 m_{2}}\right)\left(p_{1}-p_{2}\right)+\frac{1}{2}, \\
L_{2}^{L} & =1-L_{1}^{L}=\left(\frac{1}{m_{1}}-\frac{1}{2 m_{2}}\right)\left(p_{1}-p_{2}\right)+\frac{1}{2} .
\end{aligned}
$$

In case (iv), where $p_{1}-p_{2} \in\left[m_{2}, m_{1}\right]$, position $\bar{x}_{2}^{L}$ lies in $\left[x_{2}-(1 / 2), x_{1}\right]$. According to (3) and (6), equation (8) can be changed into

$$
\begin{aligned}
p_{1}-p_{2}= & k\left[\left(x_{2}-\bar{x}_{2}^{L}\right)-\frac{1}{2}\left(x_{2}-\bar{x}_{2}^{L}\right)^{2}\right] \\
& -k\left[\left(x_{1}-\bar{x}_{2}^{L}\right)-\frac{1}{2}\left(x_{1}-\bar{x}_{2}^{L}\right)^{2}\right] .
\end{aligned}
$$

Solving the equation above, we have

$$
\bar{x}_{2}^{L}=\frac{1}{2}\left(x_{1}+x_{2}\right)-\frac{p_{1}-p_{2}}{k\left(x_{1}-x_{2}\right)}-1 .
$$

Then, the lengths of attraction domains are

$$
\begin{aligned}
L_{1}^{L} & =\bar{x}_{1}^{L}-\bar{x}_{2}^{L}=1-\frac{p_{1}-p_{2}}{k\left(2+x_{1}-x_{2}\right)}+\frac{p_{1}-p_{2}}{k\left(x_{1}-x_{2}\right)} \\
& =1+\frac{2\left(p_{1}-p_{2}\right)}{k\left(x_{1}-x_{2}\right)\left(2+x_{1}-x_{2}\right)}=-\frac{1}{m_{1}}\left(p_{1}-p_{2}\right)+1, \\
L_{2}^{L} & =1-L_{1}^{L}=\frac{1}{m_{1}}\left(p_{1}-p_{2}\right) .
\end{aligned}
$$

The proof of Lemma 3. First, we prove the existence and uniqueness of the solution of (28). Noting that $A>0, C<0$, and $0<\theta_{0} \leq \theta \leq-(B / 2 A)$, it can be computed directly that

$$
\begin{aligned}
G(s, \theta)= & 12 \theta(2-s)(1+s)+4(2-s)^{2}(1+s)^{2} \\
& -9 \theta(\theta+1)(1-2 s) \\
= & -A \theta^{2}-B \theta-C \leq-A \theta_{0}^{2}-B \theta_{0}-C=0, \\
G\left(\frac{1}{2}, \theta\right)= & 27 \theta+\frac{81}{4}>0 .
\end{aligned}
$$

It follows from intermediate zero theorem for continuous functions that there exists $x^{*} \in[s,(1 / 2)]$ satisfying (28). In addition, since $(\partial G / \partial x)=12(1-2 x)+8(2-x)$ $(1+x)(1-2 x)+18 \theta(\theta+1)>0$, the solution $x^{*}$ is unique.

Second, we compute the solution $x^{*}$ by Ferrari method for quartic equation. Employing variable transformation $p=(1 / 2)-x$, equation $(28)$ can be written as

$$
y^{4}+a y^{2}+b y+c=0
$$

Completing the square yields

$$
\left(y^{2}+a+\eta\right)^{2}=(2 \eta+a) y^{2}-b y+\eta^{2}+2 a \eta+a^{2}-c .
$$

By Cardano's formula, we see that $\eta$ satisfies the following cubic equation:

$$
\left(\eta+\frac{5}{6} a\right)^{3}+\alpha\left(\eta+\frac{5}{6} a\right)+\beta=0
$$

which is equivalent to

$$
\eta^{2}+2 a \eta+a^{2}-c=\frac{b^{2}}{4(2 \eta+a)} .
$$

Combining with (A.17) and (A.19), we see that

$$
\left(y^{2}+h\right)^{2}=(\xi y+\zeta)^{2} \text {. }
$$

For this equation, there are four roots:

$$
\begin{aligned}
y_{1,2} & =\frac{\xi \pm \sqrt{\xi^{2}-4(h-\zeta)}}{2} \\
y_{3,4} & =\frac{-\xi \pm \sqrt{\xi^{2}-4(h+\zeta)}}{2}
\end{aligned}
$$

where $y_{2}=\left(\xi-\sqrt{\xi^{2}-4(h-\zeta)} / 2\right)$ lies in $[0,(1 / 2)]$. Therefore, $x^{*}$ defined in (29) is in $[s,(1 / 2)]$ and it is the solution of (28).

Finally, we prove that $\left(\mathrm{d} x^{*} / \mathrm{d} \theta\right)>0$. Let $\widetilde{A}=9(1-2 x)$, $\widetilde{B}=9(1-2 x)-12(2-x)(1+x), \quad$ and $\widetilde{C}=-4(2-x)^{2}$ $(1+x)^{2}$. It follows from (28) that $\theta=\left(-\widetilde{B}+\sqrt{\widetilde{B}^{2}-4 \widetilde{A} \widetilde{C}} / 2 \widetilde{A}\right)$. It is computed directly that $(\partial G / \partial x)=12(1-2 x)+8(2-x)(1+x)(1-2 x)+18 \theta(\theta+$ 1) $>0$ and

$$
\begin{aligned}
(\partial G / \partial \theta) & =12(2-x)(1+x)-9(2 \theta+1)(1-2 x) \\
& =-\sqrt{\widetilde{B}^{2}-4 \widetilde{A} \widetilde{C}}<0 .
\end{aligned}
$$

Therefore, $\left(\mathrm{d} x^{*} / \mathrm{d} \theta\right)=-((\partial G / \partial \theta) /(\partial G / \partial x))>0$.

\section{Data Availability}

The data used to support the findings of this study are available from the corresponding author upon request.

\section{Conflicts of Interest}

The authors declare that they have no conflicts of interest.

\section{Acknowledgments}

This work was supported in part by the Basic and Frontier Research Projects of Chongqing (Nos. cstc2018jcyjAX0606 and cstc2020jsyj-zdxwtBX0003), in part by the Science and Technology Research Program of Chongqing Municipal 
Education Commission (No. KJQN201900701), in part by the Chinese National Social Science Foundation (No. 18XGL006), in part by Chongqing Social Science Planning Priorities Project (No. 2020TBWT-ZD002), in part by the team building project for Graduate Tutors in Chongqing (No. JDDSTD201802), and in part by the Promotion Project for Young and Middle-aged Teachers' Basic Scientific Research Ability in Colleges and Universities of Guangxi (No 2019KY0382).

\section{References}

[1] J. von Neumann, "Zur theorie der gesellschaftsspiele," Mathematische Annalen, vol. 100, no. 1, pp. 295-320, 1928.

[2] T. Ichiishi, A. Neyman, Y. Tauman, and K. Shell, Game Theory and Applications, Economic Theory, Econometrics, and Mathematical Economics, Elsevier Inc, Amsterdams, Netherlands, 1990.

[3] V. M. Bier and M. N. Azaiez, Game Theoretic Risk Analysis of Security Threats, Springer, Berlin, Germany, 2009.

[4] S. Lasaulce and H. Tembine, Game Theory and Learning for Wireless Networks. Fundamentals and Applications, Academic Press, Cambridge, MA, USA, 2011.

[5] J. Chen and B. Chen, "When should the offline retailer implement price matching?," European Journal of Operational Research, vol. 277, no. 3, pp. 996-1009, 2019.

[6] R. M. Braid, "The locations of firms on intersecting roadways," The Annals of Regional Science, vol. 50, no. 3, pp. 791-808, 2013.

[7] Q. Gong, Q. Liu, and Y. Zhang, "Optimal product differentiation in a circular model," Journal of Economics, vol. 119, no. 3, pp. 219-252, 2016.

[8] E. Talamàs, "Price dispersion in stationary networked markets," Games and Economic Behavior, vol. 115, pp. 247-264, 2019.

[9] R. Biscaia and I. Mota, "Models of spatial competition: a critical review," Papers in Regional Science, vol. 92, no. 4, pp. 851-871, 2013.

[10] W.-C. Guo, F.-C. Lai, and D.-Z. Zeng, "A Hotelling model with production," Mathematical Social Sciences, vol. 73, pp. 40-49, 2015.

[11] D. Pal and J. Sarkar, "Spatial cournot competition among multi-plant firms in a circular city," Southern Economic Journal, vol. 73, no. 1, pp. 246-258, 2006.

[12] J.-F. Tsai and F.-C. Lai, "Spatial duopoly with triangular markets," Papers in Regional Science, vol. 84, no. 1, pp. 47-59, 2005.

[13] B. Pelegrín, P. Fernández, and M. D. García, "Computation of multi-facility location Nash equilibria on a network under quantity competition," Networks and Spatial Economics, vol. 18, no. 4, pp. 999-1017, 2018.

[14] H. Hotelling, "Stability in competition," The Economic Journal, vol. 39, no. 153, pp. 41-57, 1929.

[15] C. d'Aspremont, J. J. Gabszewicz, and J.-F. Thisse, "Computation of multi-facility location nash equilibria on a network under quantity competition," Econometrica, vol. 47, no. 4, pp. 1145-1150, 1979.

[16] A. P. Lerner and H. W. Singer, "Some notes on duopoly and spatial competition," Journal of Political Economy, vol. 45, no. 2, pp. 145-186, 1937.

[17] A. Smithies, "Optimum location in spatial competition," Journal of Political Economy, vol. 49, no. 3, pp. 423-439, 1941.
[18] D. Graitson, "Spatial competition a la Hotelling: a selective survey," The Journal of Industrial Economics, vol. 31, no. 1/2, pp. 11-25, 1982.

[19] N. Economides, "Minimal and maximal product differentiation in Hotelling's duopoly," Economics Letters, vol. 21, no. 1, pp. 67-71, 1986.

[20] J. H. Hamilton, J.-F. Thisse, and A. Weskamp, "Spatial discrimination," Regional Science and Urban Economics, vol. 19, no. 1, pp. 87-102, 1989.

[21] S. Brenner, "Hotelling games with three, four, and more players," Journal of Regional Science, vol. 45, no. 4, pp. 851-864, 2005.

[22] M. Peitz, "The circular road revisited: uniqueness and supermodularity," Research in Economics, vol. 53, no. 4, pp. 405-420, 1999.

[23] M. A. de Frutos, H. Hamoudi, and X. Jarque, "Equilibrium existence in the circle model with linear quadratic transport cost," Regional Science and Urban Economics, vol. 29, no. 5, pp. 605-615, 1999.

[24] W.-C. Guo and F.-C. Lai, "Spatial Cournot competition in a linear-circular market," The Annals of Regional Science, vol. 54, no. 3, pp. 819-834, 2015.

[25] B. Gupta, F.-C. Lai, D. Pal, J. Sarkar, and C.-M. Yu, "Where to locate in a circular city?," International Journal of Industrial Organization, vol. 22, no. 6, pp. 759-782, 2004.

[26] W.-C. Guo and F.-C. Lai, "Spatial cournot competition in two intersecting circular markets," The Annals of Regional Science, vol. 64, no. 1, pp. 37-56, 2020.

[27] Y. Chen and M. H. Riordan, "Price and variety in the spokes model," The Economic Journal, vol. 117, no. 522, pp. 897-921, 2007.

[28] M. E. Sáiz and E. M. T. Hendrix, "Methods for computing Nash equilibria of a location-quantity game," Computers \& Operations Research, vol. 35, no. 10, pp. 3311-3330, 2008.

[29] P. J. Lederer and A. P. Hurter, "Competition of firms: discriminatory pricing and location," Econometrica, vol. 54, no. 3, pp. 623-640, 1986.

[30] S. C. Salop, "Monopolistic competition with outside goods," The Bell Journal of Economics, vol. 10, no. 1, pp. 141-156, 1979. 